\title{
Mango and COVID-19: The impact on and coping of Namdokmai Sithong mango export farmers in Khon Kaen, Thailand during the pandemic of COVID-19
}

\author{
Patcharaporn Sornsena ${ }^{1,3}$, Kanlaya Mikhama ${ }^{2}$, and Yos Borisutdhi ${ }^{3, *}$ \\ 1 Buri Ram Plant Propagation Center, Department of Agricultural Extension, Ministry of Agriculture and \\ Cooperatives, Buri Ram 31110, Thailand \\ 2 Faculty of Agriculture and Technology, Nakhon Phanom University, Nakhon Phanom 48000, Thailand \\ 3 Faculty of Agriculture, Khon Kaen University, Khon Kaen 40002, Thailand \\ * Correspondence author: yosboris@kku.ac.th, yospure@gmail.com; Tel.: +66-85-656-6155
}

\begin{abstract}
The pandemic of COVID-19 has had impacts on mango export of farmers and they must have coping strategies to survival. Ban Haet Mango Export Community Enterprise is a leading community enterprise that exports Namdokmai Sithong mango. Since 2007, the enterprise has followed the standards set by the Good Agricultural Practices (GAP) in four season per year, ensuring high quality of their mangoes fit for sales in export and domestic markets. For exporting, sold premium graded to distributors and export to Japan, South Korea and China. A few premium graded and a graded lower were sold in the domestic market by farmer themselves and via middlemen. However, at the end of the year 2019, the COVID-19 epidemic erupted, which later spread across the globe that heavily affected on the mango market of farmers. The severity of the impact was identified in two phases, the 1st period during 10-24 March 2020 and the 2nd period during 25 March 31 May 2020. It was found that three main partners have suffered and their coping strategies were divided into the following two periods: (1) Impact on members and their coping strategies; The $1^{\text {st }}$ period, can't sell mangoes and they put on hold the harvesting of the produce and diverted manpower for finding new markets. The $2^{\text {nd }}$ period, coordinate domestic marketing channel support with relevant sectors, they were able to sell some of their produce. (2) Impact on the community enterprise and its coping strategies; The $1^{\text {st }}$ period, the enterprise was not able to gather the produce from the members for distribution. They coordinated marketing channel support with relevant sectors. The 2 nd period, they could gather some of the produce with price slashed by forty percent for export and fifty percent for domestic market when compared with previous year. (3) Impact on export distributors and their adjustment. The $1^{\text {st }}$ period, they were heavily impacted being unable to buy the produce from the enterprise for export. They looked for ways to buy the produce and distributed it to the international market. The $2^{\text {nd }}$ period, they were able to purchase some of the produce for export and the mangoes were transported throughout the chartered flights.
\end{abstract}

Keywords: Coronavirus disease 2019; community enterprise; mango farmer; mango marketing; coping strategy.

\section{Introduction}

A pandemic has far-reaching impacts on large sections of the world's population. Throughout history, pandemics have wreaked havoc to human activity. Some of the severe examples of the pandemics the world has experienced include the Cholera pandemic of 1816 which originated in the Indian city of Bengal, the 1904 Plague Disease (Thongcharoen, 2009a) and the Spanish Flu that lasted from 1918-1919 (Thongcharoen, 2009b). In late 2002, a SARS (Severe Acute Respiratory Syndrome) epidemic erupted in China and eventually spread to different parts of the world (Ministry of Public Health, 2011). Later, a MERS (Middle East Respiratory Syndrome) epidemic broke out in Saudi Arabia in 2012 (Danaisawat \& Hengpraprom, 2018). During the years 2004-2006, there was a spread of an avian influenza, also known as a Bird Flu (Bureau of General Communicable Diseases, 2019). Since late 2019, the spread of the COVID-19 (Coronavirus disease 2019) pandemic has caused enormous damage throughout the globe. The disease can be transmitted via direct and indirect human-to- 
human contact, for example through respiratory droplets, faeces and the touching of eyes, nose or mouth. One patient can further infect 2-4 people (Department of Disease Control, 2020) leading to a quick spread of the disease. The pandemic has impacted the delivery of foods or agricultural producers to consumers, disrupting the production chain, and has eventually led to an ongoing crisis of global recessions (Cheewatrakoolpong, 2020). For example, Ban Haet Mango Export Community Enterprise (BHME Community Enterprise), Khon Kaen, Thailand who produce Namdokmai Sithong mango (NDMST mango) were greatly impacted in the transport of produce from farms to consumers during COVID-19 crisis in March to May 2020 as the first period of pandemic in Thailand. There are also quite a few farmers or agricultural entrepreneurs in Thailand or another country has been more or less impacted by COVID-19 crisis as same as BHME Community Enterprise.

The COVID-19 pandemic originated from China at the end of the year 2019 and later spread to every corner of the globe. Chantapong et al. (2020) reported that the pandemic has heavily affected the world economy through the disruption of the supply chain as a result of shortages of labour and raw materials. The crisis is particularly damaging because of China's position as one of the world's leading producers and consumers. The spread of the disease and its impacts have sent shock waves throughout the world, disrupting international trade, tourism and services, to name just a few. The closing of international borders to slow the spread of the disease has caused strain to the food supply chains. The International Monetary Fund (IMF) estimated the economic cost of the COVID19 pandemic to be up to 9 trillion US dollars for the years 2020-2021, the value greater than the economies of Japan and Germany combined (Gopinath, 2020). The World Trade Organization (WTO) meanwhile estimated that the volume of international trade in 2020 would reduce by a third in comparison to that of the year before. If the situation persists, businesses and households would lose liquidity so much that their loan payments would be overdue risking a major financial crisis. This triple economic shock would be the first in the world history (Chantapong \& Tonghui, 2020).

In the agriculture and food sectors, at the height of the human disease pandemic, farmers suffered greatly being unable to sell their agricultural produce. Processing factories were in short supply of agricultural products or raw materials. Delivery of the produce was brought to an almost stop due to a number of factors including greatly reduced freight traffic and disruption on the logistics. The COVID-19 crisis is much more severe than the food crisis of 2007-2008, where supply of the main products was not heavily impacted. Since the start of the current pandemic, countries have fought hard to maintain the food supply chain.

Like anywhere else, Thailand has suffered heavily as a result of the COVID-19 pandemic, there is a huge drop in the number of international travellers and household consumption particularly during the height of the crisis in early 2020 went down sharply, partly because the "staying at home and social distancing" measures (BBC NEWS Thai, 2020). The ban on assembly of people and normal human activity including the closing of markets and restaurants as well as the sales ban of some goods affected the retail and export sectors. The electronic and car parts sectors were severely impacted. The total export of agricultural products was greatly reduced. Private consumption tended to limit to basic staple foods. The change in the behaviour of consumers where online shopping is becoming a norm has also impacted the uncertainty in the demand (Thailand Science Research and Innovation, 2020).

NDMST mango is one of Thailand's export fruits that has suffered from the impact of the COVID19 pandemic. Its export to Japan and South Korea was heavily disrupted. The reduced number of operational flights by commercial airlines, the increased cost for transport, and the price drop in the country have incurred heavy losses to the NDMST mango farmers (Thailand Science Research and Innovation, 2020).

According to a preliminary study, the export volume of the NDMST mango reached 12,000 tonnes in 2019 (Srinil, 2020). The province of Khon Kaen counts among the major producers of the NDMST mango around 300 tonnes per season (300 tonnes*4 seasons=1,200 tonnes per year) or with around $10 \%$ of the total export coming from Khon Kaen. Exporting the NDMST mango from Khon Kaen is managed by the BHME Community Enterprise, one of Thailand's leading community enterprises that export the mango and a sole community enterprise to export the mango in Khon Kaen. The community enterprise is certified with GAP (Good Agricultural Practices) ensuring high 
quality mangoes that can be sold both in the international and national markets. Their success led us to research questions of individual learning of the farmers, knowledge management of the BHME Community Enterprise that ensured smooth success of the export of the NDMST mango. However, because of the spread of the disease, our study was halted and did not follow the original research plan. Topics that have been covered include the condition of operation and production of the NDMST mango for the export purpose and the GAP in producing the mango for export, whereas the topics of individual learning of the enterprise members and knowledge management of the enterprise could not be carried out due to the government's measures that aimed to tackle the spread of the disease. The direction of our research however had changed course as a result and in response to the eruption of the pandemic of COVID-19 in end period the of 2019. New research questions had arisen how the members of the community enterprise adapted themselves to cope within the face of the pandemic of COVID-19.

In our farming, coping refers to the thought and actions farmer households that have to deal with during a threatening situation (Centre for Studies on Human Stress, 2019) and also coping strategies are as numerous and varied as the stressors and or impacts that precede them (Blum et al, 2012). Therefore, this study focused on the impact on and coping of NDMST mango export farmers in Khon Kaen that was carried out in March to May 2020 at the first period and high pandemic of COVID-19 in Thailand.

\section{Materials and Methods}

\subsection{Study site}

The province of Khon Kaen was chosen for this study because it has farmers that grow the NDMST mango for export, which is managed by the BHME Community Enterprise. The community enterprise is the only one in Khon Kaen to export the NDMST mango and counts as one of the most important mango production community enterprises in Thailand. Its production of the mango in the study site has been certified with GAP guaranteeing the quality of the mango fit for consumption in the domestic market as well as for export and during the COVID-19 pandemic in March to May 2020, the farmers were affected in exports.

\subsection{Study process}

The study was divided into three phases as follows.

Phase 1: Study of the Context and General Management of the BHME Community Enterprise Studied the context as well as the for-export-purpose NDMST mango production management of the BHME Community Enterprise by creating sub-topics that served as a tool for secondary data collection by reviewing the literature and primary data collection by observing and interviewing key informants such as the chairman, committee and farmer leader of the BHME Community Enterprise as well as government officers. The data were then analysed using the $6 \mathrm{C}$ analysis beginning with analysis while data collection to be followed by categorization, comparison, correlation, construction and creation (Borisutdhi, 2015).

Phase 2: Study of the production management and GAP application in the NDMST mango production for export by members of the BHME Community Enterprise - Results from Phase 1 were used to form the basis of questionnaire. Sixty-three members of the community enterprise were asked. The data was then analysed using descriptive statistics and Cross-tab analysis.

Phase 3: Study of the impact on and coping towards NDMST mango production for export by the BHME Community Enterprise and its members during the COVID-19 pandemic - Sub-topics were created as a tool for data collection. Secondary data was collected through the review of involved documents while primary data collection was done by observing and interviewing key informants such as the chairman, committee and farmer leader of the BHME Community Enterprise as well as government officers. The data were then analysed using the $6 \mathrm{C}$ analysis.

The data were analysed by using the $6 \mathrm{C}$ analysis in Phase 1 and 3; each phase started from data analysis while data collection ( $\mathrm{C} 1$ ) (were analysed which were then answered by the farmer, and during analysis of the answers, questions were continued by the researcher) was to be followed by data categorization (C2) of each issue and after that the analysis was carried out by comparison (C3) and correlation (C4) for each issue. All of them were integrated into knowledge construction (C5) (such as Figure 
2, 3 and 4) and accuracy of the results was again rechecked with farmer leaders or data sources, and creation (C6) analysis was performed for discussion and recommendation based on the findings.

\section{Results and Discussion}

\subsection{Context and general management of the BHME community enterprise}

\subsubsection{Context of the BHME community enterprise}

The enterprise encompassed 63 members and area of 1,460 rai (6.25 rai=1 hectare) for the NDMST mango production, 975 rai of which had already yielded mangos while the rest had not yielded. The area lies in the provinces of Khon Kaen, Nakhon Ratchasima and Maha Sarakham. The enterprise and its members are supported by the Farmers Institute, the Farmer Leader and related agencies concerning the mango production that meets the requirements of the GAP in order to ensure good production quality and efficient management and operation (Figure 2 and Table 1).

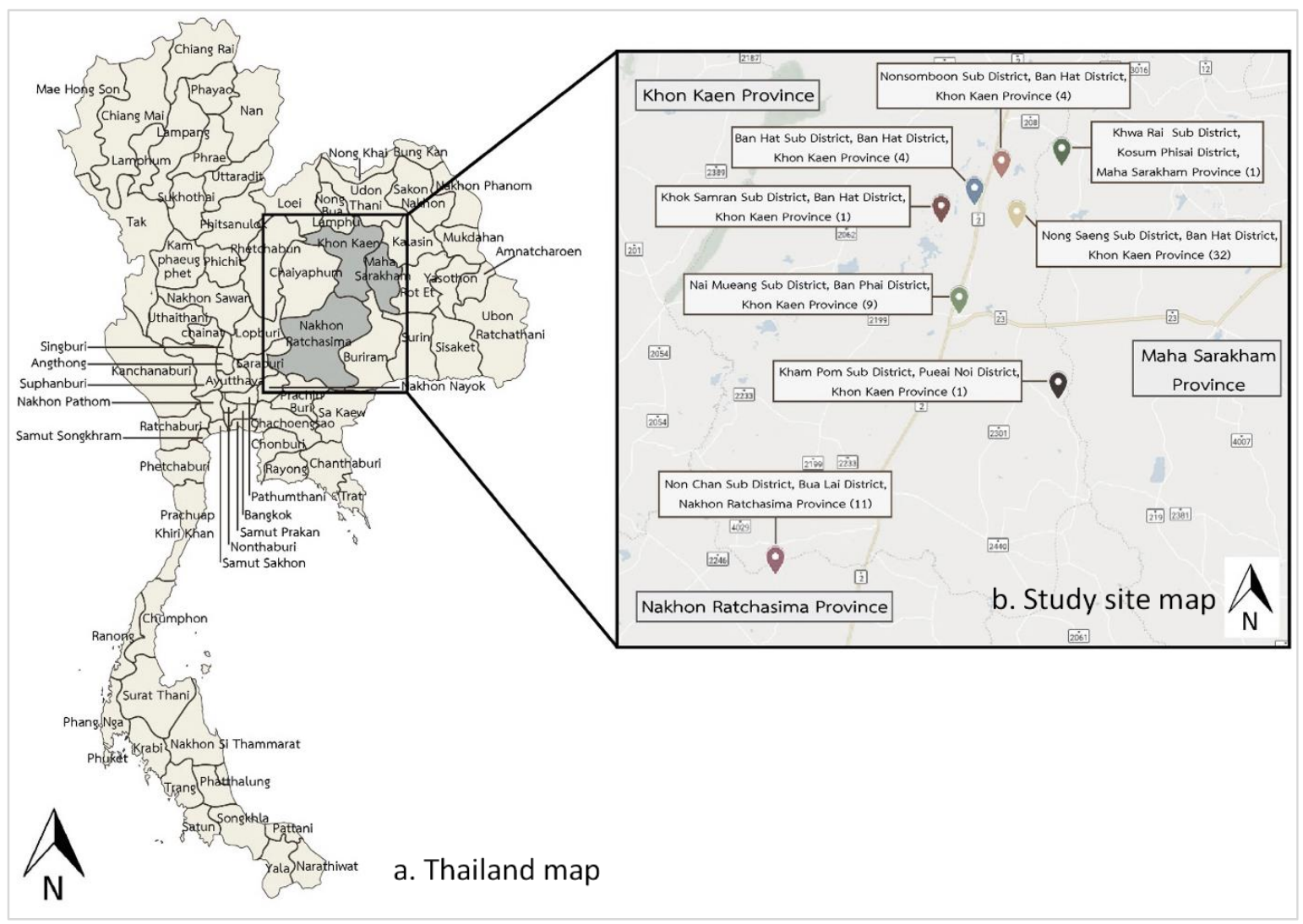

Figure 1. Map showing the distribution of members of the BHME community enterprise

Table 1. Socio-economic context of member of the BHME community enterprise

\begin{tabular}{|c|c|c|c|c|}
\hline Socio-economic context of member & $N(63)$ & $\%$ & Average $\pm \sigma$ & Range \\
\hline 1. Age (year) & & & $51.29 \pm 10.959$ & $79-26$ \\
\hline \multicolumn{5}{|l|}{ 2. Education level } \\
\hline 2.1 Lower than primary school & 2 & 3.17 & & \\
\hline 2.2 Early primary school & 15 & 23.81 & & \\
\hline 2.3 Primary school & 17 & 27.98 & & \\
\hline 3.4 Early secondary school & 9 & 14.29 & & \\
\hline $\begin{array}{l}\text { 2.4 Secondary school or vocational } \\
\text { certification }\end{array}$ & 12 & 19.05 & & \\
\hline $\begin{array}{l}\text { 2.6 Vocational diploma or associate } \\
\text { degree }\end{array}$ & 4 & 6.35 & & \\
\hline 2.7 Bachelor degree & 3 & 4.76 & & \\
\hline 2.8 Higher than bachelor degree & 1 & 1.59 & & \\
\hline GAP certification & & & & \\
\hline 3.1 GAP certification for individual & 51 & 71.83 & & \\
\hline
\end{tabular}




\begin{tabular}{|c|c|c|c|c|c|}
\hline & Socio-economic context of member & $N(63)$ & $\%$ & Average $\pm \sigma$ & Range \\
\hline & 3.2 GAP certification for group & 14 & 19.72 & & \\
\hline & 3.3 Not yet GAP certification & 6 & 8.45 & & \\
\hline 4 & Harvested area (rai (6.25 rai=1 hectare) ) & & & $17.40 \pm 13.255$ & $60-1$ \\
\hline 5 & $\begin{array}{l}\text { New plantation area, not yet harvesting } \\
\text { (rai) }\end{array}$ & & & $11.54 \pm 7.235$ & $31-2$ \\
\hline 6 & Experience in mango production (year) & & & $11.38 \pm 7.786$ & $31-2$ \\
\hline 7 & Members in community enterprise (year) & & & $8.46 \pm 6.635$ & $20-1$ \\
\hline 8 & $\begin{array}{l}\text { On-agricultural sector income (Thai Baht } \\
\text { (THB per year)) }\end{array}$ & & & $26,0571.43 \pm 270,166.718$ & $1,200,000-6,000$ \\
\hline 9 & $\begin{array}{l}\text { Off-agricultural sector income (THB per } \\
\text { year) }\end{array}$ & & & $92,080 \pm 104,435.754$ & $500,000-5,000$ \\
\hline 10 & Mango production income (THB per year) & & & $192,196.43 \pm 242,470.048$ & $1,000,000-5,000$ \\
\hline 11 & $\begin{array}{l}\text { Agriculture labour number in farm household } \\
\text { (person) }\end{array}$ & & & $2.63 \pm 1.168$ & $6-1$ \\
\hline 12 & Hired labour (some time) (person) & & & $3.75 \pm 2.708$ & $13-1$ \\
\hline
\end{tabular}

\subsubsection{The general management of BHME community enterprise}

The BHME enterprise was found to have the following key points for their operation. (1) Benefit management: profits were deducted to the fund set up for the administration and management of the enterprise. (2) Production management: quality control started from the beginning of the production all through to the harvest season. Characters such as colour and shape of the fruit, and ripeness, which should be between $85-90 \%$, were controlled and another wise production planning based on marketing demand. (3) Marketing management and processing to value added: (a) international market - export companies notified the enterprise of the amount of the produce they needed and the enterprise then collected the mangos to be delivered to the companies. The companies sorted the mangos (only those graded $3 \mathrm{~A}$ or $2 \mathrm{~A}$ were exported), packaged and delivered them. Major export markets in order of the sales volume were Japan, South Korea and China. (b) domestic market and processing to value added mangos that were graded lower than $3 \mathrm{~A}$ or $2 \mathrm{~A}$ were sold in the domestic market via middlemen who sorted the mangos into type No.0, No.1 and No.2, packaged and delivered them within wholesale and modern trade market. The enterprise also sold the mangos themselves and so did individual members. Some of the premium $3 \mathrm{~A}$ and $2 \mathrm{~A}$ mangos were packaged and sold by the enterprise in the domestic market. Moreover, products were processed to add value to them. All of these served as advertisement channels for the community enterprise and its members (Figure 2).

According to our study, the BHME Community Enterprise was efficiently operated, meeting the demand of the export companies as well as being able to distribute their mangos within the domestic market. What was found in this study agreed well with Dangbudda's observations (Dangbudda, 2011) that success of the community enterprises depended on a number of factors namely: quality of the NDMST mango that met the standards; management based on efficient system of management and division of work structure; development of the mangos and their packaging; distribution of the product in the local, domestic and export markets; increasing demand; potential of group leader to facilitate matters both within and external to the community enterprise; and finance in which members contributed their own funds to the production of the NDMST mango. All these factors contributed to the growth of the BHME Community Enterprise. Jumroensarn (2010) reported that the NDMST mango was distributed through the following five channels; direct distribution to consumers, through middlemen who collected the mangos for wholesale and retail sellers, through wholesale sellers who then distributed to retail sellers, by export companies who distributed the mangos to foreign consumers, and from retail sellers to consumers. The marketing cost was highest among the retail sellers, followed by the wholesale sellers and the collection sellers. 


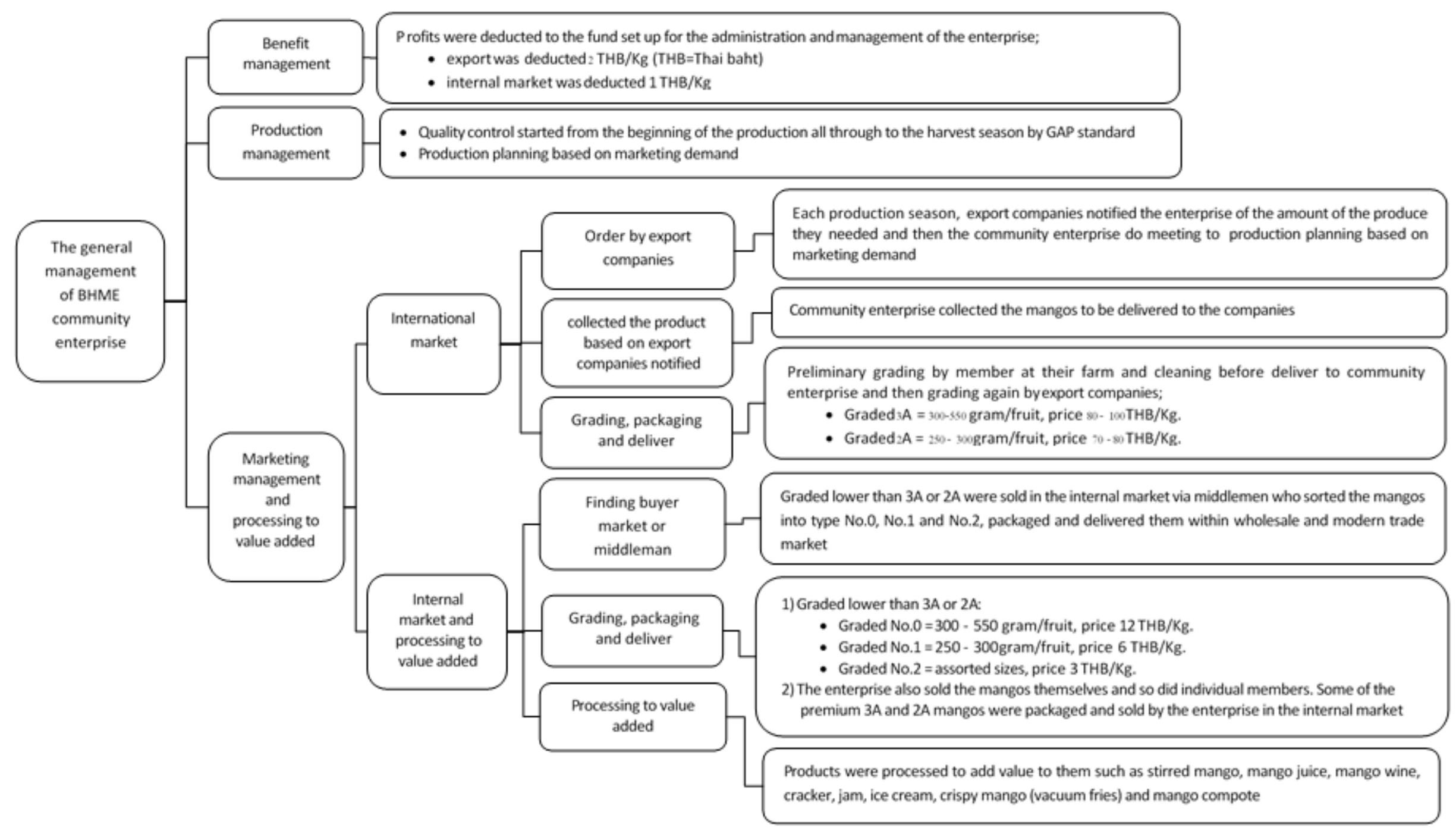

Figure 2. The general management of BHME community enterprise in 2015-2019 (before the pandemic of COVID-19) 
Table 2. GAP application in the NDMST mango production by members of the BHME community enterprise (before the pandemic of COVID-19)

\begin{tabular}{|c|c|c|c|c|c|c|c|c|c|c|c|c|c|c|c|c|c|c|c|c|}
\hline \multirow[b]{2}{*}{ Module } & \multicolumn{4}{|c|}{ Members in community enterprise (year) } & \multicolumn{4}{|c|}{ Experience in mango production (year) } & \multicolumn{4}{|c|}{ Harvesting farm area (rai) } & \multicolumn{4}{|c|}{ Agriculture labour number in farm household (person) } & \multicolumn{4}{|c|}{ Mango production income (Thai Baht (THB)) } \\
\hline & $\begin{array}{l}\leq 5 \text { year } \\
\text { (Avg士o) } \\
\text { (Means) }\end{array}$ & \begin{tabular}{|l|}
$6-10$ year \\
(Avg $\pm \sigma)$ \\
(Means) \\
\end{tabular} & \begin{tabular}{|c|}
$11-15$ year \\
(Avg士o) \\
(Means)
\end{tabular} & \begin{tabular}{|l|}
$\geq 16$ year \\
(Avg士o) \\
(Means)
\end{tabular} & $\begin{array}{l}\leq 5 \text { year } \\
\text { (Averageto) } \\
\text { (Means) }\end{array}$ & $\begin{array}{l}\text { 6-10 year } \\
\text { (Averageto) } \\
\text { (Means) }\end{array}$ & \begin{tabular}{|c}
$11-15$ year \\
(Averageto) \\
(Means)
\end{tabular} & \begin{tabular}{|c|}
$\geq 16$ year \\
(Averageto) \\
(Means)
\end{tabular} & \begin{tabular}{|c} 
Not yet \\
(Averageto) \\
(Means)
\end{tabular} & \begin{tabular}{|c|} 
5) \\
(Averaget-10 rai \\
(Means)
\end{tabular} & $\begin{array}{l}11-20 \text { rai } \\
\text { (Averageto) } \\
\text { (Means) }\end{array}$ & $\begin{array}{c}\geq 21 \text { rai } \\
\text { (Averageto) } \\
\text { (Means) }\end{array}$ & \begin{tabular}{|c|}
1 person \\
(Averageto) \\
(Means)
\end{tabular} & \begin{tabular}{|c|}
2 person \\
(Averageto) \\
(Means)
\end{tabular} & \begin{tabular}{|c} 
person \\
(Averageto) \\
(Means)
\end{tabular} & \begin{tabular}{|l}
$\geq 4$ person \\
(Averageto) \\
(Means)
\end{tabular} & \begin{tabular}{|c|} 
Not yet \\
(Averageto) \\
(Means)
\end{tabular} & \begin{tabular}{|c|}
$0.01-$ \\
100,000 THB \\
(Averageto) \\
(Means)
\end{tabular} & \begin{tabular}{|c|}
$100,001-$ \\
200,000 \\
THB \\
(Averageto) \\
(Means) \\
\end{tabular} & $\begin{array}{c}2200,001 \text { THB } \\
\text { (Average } \pm \sigma \text { ) } \\
\text { (Means) }\end{array}$ \\
\hline $\begin{array}{l}\text { 1. Water resource } \\
\text { management }\end{array}$ & $\begin{array}{c}2.92 \pm 0.272 \\
\text { ST }\end{array}$ & $\begin{array}{c}2.95 \pm 0.120 \\
\text { ST }\end{array}$ & $\begin{array}{c}3.00 \pm 0.000 \\
\text { ST }\end{array}$ & $\begin{array}{c}2.97 \pm 0.075 \\
\text { ST }\end{array}$ & $\begin{array}{c}2.96 \pm 0.155 \\
\text { ST }\end{array}$ & $\begin{array}{c}2.97 \pm 0.095 \\
\text { ST }\end{array}$ & $\begin{array}{c}3.00 \pm 0.000 \\
\text { ST }\end{array}$ & $\begin{array}{c}2.88 \pm 0.340 \\
\text { ST }\end{array}$ & $\begin{array}{c}2.89 \pm 0.227 \\
\text { ST }\end{array}$ & $\begin{array}{c}3.00 \pm 0.000 \\
\text { ST }\end{array}$ & $\begin{array}{c}2.97 \pm 0.138 \\
\text { ST }\end{array}$ & $\begin{array}{c}2.88 \pm 0.294 \\
\text { ST }\end{array}$ & $\mid \begin{array}{c}3.00 \pm 0.000 \\
\text { ST }\end{array}$ & \begin{tabular}{|c|}
$2.91 \pm 0.274$ \\
ST
\end{tabular} & $\begin{array}{c}3.00 \pm 0.000 \\
\text { ST }\end{array}$ & $\begin{array}{c}2.98 \pm 0.055 \\
\text { ST }\end{array}$ & $\begin{array}{c}3.00 \pm 0.000 \\
\text { ST }\end{array}$ & $\begin{array}{c}2.95 \pm 0.134 \\
\text { ST }\end{array}$ & $\begin{array}{c}2.85 \pm 0.401 \\
\text { ST }\end{array}$ & $\begin{array}{c}3.00 \pm 0.000 \\
\text { ST }\end{array}$ \\
\hline $\begin{array}{c}\text { 2. Farm land and } \\
\text { cultiation } \\
\text { management }\end{array}$ & $\begin{array}{c}2.98 \pm 0.108 \\
\text { ST }\end{array}$ & $\begin{array}{c}2.94 \pm 0.171 \\
\text { ST }\end{array}$ & $\begin{array}{c}3.00 \pm 0.000 \\
\text { ST }\end{array}$ & $\begin{array}{c}3.00 \pm 0.000 \\
\text { ST }\end{array}$ & $\begin{array}{c}3.00 \pm 0.000 \\
\text { ST }\end{array}$ & $\begin{array}{c}2.95 \pm 0.166 \\
\text { ST }\end{array}$ & $\begin{array}{c}3.00 \pm 0.000 \\
\text { ST }\end{array}$ & $\begin{array}{c}2.99 \pm 0.049 \\
\text { ST }\end{array}$ & $\begin{array}{c}3.00 \pm 0.000 \\
\text { ST }\end{array}$ & $\begin{array}{c}3.00 \pm 0.000 \\
\text { ST }\end{array}$ & $\begin{array}{c}2.97 \pm 0.138 \\
\text { ST }\end{array}$ & $\begin{array}{c}2.97 \pm 0.127 \\
\text { ST }\end{array}$ & $\begin{array}{c}3.00 \pm 0.000 \\
\text { ST }\end{array}$ & $\begin{array}{c}2.97 \pm 0.118 \\
\text { ST }\end{array}$ & $\begin{array}{c}2.96 \pm 0.155 \\
\text { ST }\end{array}$ & $\begin{array}{c}3.00 \pm 0.000 \\
\text { ST }\end{array}$ & $\begin{array}{c}3.00 \pm 0.000 \\
\text { ST }\end{array}$ & $\begin{array}{c}2.96 \pm 0.148 \\
\text { ST }\end{array}$ & $\begin{array}{c}2.98 \pm 0.058 \\
\text { ST }\end{array}$ & $\begin{array}{c}3.00 \pm 0.000 \\
\text { ST }\end{array}$ \\
\hline $\begin{array}{l}\text { 3. Agricultural } \\
\text { hazardous } \\
\text { substances } \\
\text { management }\end{array}$ & $\begin{array}{c}2.96 \pm 0.095 \\
\text { ST }\end{array}$ & $\begin{array}{c}2.95 \pm 0.088 \\
\text { ST }\end{array}$ & $\begin{array}{c}2.97 \pm 0.082 \\
\text { ST }\end{array}$ & $\begin{array}{c}2.98 \pm 0.055 \\
\text { ST }\end{array}$ & $\begin{array}{c}2.97 \pm 0.070 \\
\text { ST }\end{array}$ & $\begin{array}{c}2.95 \pm 0.105 \\
\text { ST }\end{array}$ & $\begin{array}{c}2.97 \pm 0.082 \\
\text { ST }\end{array}$ & $\begin{array}{c}2.98 \pm 0.066 \\
\text { ST }\end{array}$ & $\begin{array}{c}2.94 \pm 0.098 \\
\text { ST }\end{array}$ & $\begin{array}{c}3.00 \pm 0.000 \\
\text { ST }\end{array}$ & $\begin{array}{c}2.95 \pm 0.112 \\
\text { ST }\end{array}$ & $\begin{array}{c}2.97 \pm 0.068 \\
\text { ST }\end{array}$ & $\begin{array}{c}2.95 \pm 0.088 \\
\text { ST }\end{array}$ & $\begin{array}{c}2.98 \pm 0.063 \\
\text { ST }\end{array}$ & $\begin{array}{c}2.97 \pm 0.103 \\
\text { ST }\end{array}$ & $\begin{array}{c}2.94 \pm 0.096 \\
\text { ST }\end{array}$ & $\begin{array}{c}3.00 \pm 0.000 \\
\text { ST }\end{array}$ & $\begin{array}{c}2.96 \pm 0.098 \\
\text { ST }\end{array}$ & $\begin{array}{c}2.97 \pm 0.078 \\
\text { ST }\end{array}$ & $\begin{array}{l}2.97 \pm 0.078 \\
\text { ST }\end{array}$ \\
\hline $\begin{array}{l}\text { 4. Quality } \\
\text { management in } \\
\text { farming and } \\
\text { before } \\
\text { harvesting } \\
\text { processes }\end{array}$ & $\begin{array}{c}2.99 \pm 0.036 \\
\text { ST }\end{array}$ & $\begin{array}{c}2.94 \pm 0.126 \\
\text { ST }\end{array}$ & $\begin{array}{c}2.97 \pm 0.082 \\
\text { ST }\end{array}$ & $\begin{array}{c}3.00 \pm 0.000 \\
\text { ST }\end{array}$ & $\begin{array}{c}2.99 \pm 0.052 \\
\text { ST }\end{array}$ & $\begin{array}{c}2.97 \pm 0.095 \\
\text { ST }\end{array}$ & $\begin{array}{c}2.97 \pm 0.082 \\
\text { ST }\end{array}$ & $\begin{array}{c}3.00 \pm 0.000 \\
\text { ST }\end{array}$ & $\begin{array}{c}2.91 \pm 0.157 \\
\text { ST }\end{array}$ & $\begin{array}{c}3.00 \pm 0.000 \\
\text { ST }\end{array}$ & $\begin{array}{c}2.98 \pm 0.092 \\
\text { ST }\end{array}$ & $\begin{array}{c}2.98 \pm 0.068 \\
\text { ST }\end{array}$ & $\begin{array}{c}2.98 \pm 0.055 \\
\text { ST }\end{array}$ & $\begin{array}{c}2.99 \pm 0.038 \\
\text { ST }\end{array}$ & $\begin{array}{c}3.00 \pm 0.000 \\
\text { ST }\end{array}$ & $\begin{array}{c}2.97 \pm 0.075 \\
\text { ST }\end{array}$ & $\begin{array}{c}3.00 \pm 0.000 \\
\text { ST }\end{array}$ & $\begin{array}{c}2.97 \pm 0.090 \\
\text { ST }\end{array}$ & $\begin{array}{c}2.98 \pm 0.058 \\
\text { ST }\end{array}$ & $\begin{array}{c}3.00 \pm 0.000 \\
\text { ST }\end{array}$ \\
\hline $\begin{array}{l}\text { 5. Quality } \\
\text { management in } \\
\text { harvesting and } \\
\text { post havesting } \\
\text { processes }\end{array}$ & $\begin{array}{c}2.99 \pm 0.036 \\
\text { ST }\end{array}$ & $\begin{array}{c}3.00 \pm 0.000 \\
\text { ST }\end{array}$ & $\begin{array}{c}3.00 \pm 0.000 \\
S T\end{array}$ & $\begin{array}{c}3.00 \pm 0.000 \\
\text { ST }\end{array}$ & $\begin{array}{c}2.99 \pm 0.052 \\
\text { ST }\end{array}$ & $\begin{array}{c}3.00 \pm 0.000 \\
\text { ST }\end{array}$ & $\begin{array}{c}3.00 \pm 0.000 \\
\text { ST }\end{array}$ & $\begin{array}{c}3.00 \pm 0.000 \\
\text { ST }\end{array}$ & $\begin{array}{c}3.00 \pm 0.000 \\
\text { ST }\end{array}$ & $\begin{array}{c}3.00 \pm 0.000 \\
\text { ST }\end{array}$ & $\begin{array}{c}3.00 \pm 0.000 \\
\text { ST }\end{array}$ & $\begin{array}{c}2.99 \pm 0.041 \\
\text { ST }\end{array}$ & $\begin{array}{c}3.00 \pm 0.000 \\
\text { ST }\end{array}$ & $\begin{array}{c}3.00 \pm 0.000 \\
\text { ST }\end{array}$ & $\begin{array}{c}3.00 \pm 0.000 \\
\text { ST }\end{array}$ & $\begin{array}{c}2.98 \pm 0.055 \\
\text { ST }\end{array}$ & $\begin{array}{c}3.00 \pm 0.000 \\
\text { ST }\end{array}$ & $\begin{array}{c}2.99 \pm 0.035 \\
\text { ST }\end{array}$ & $\begin{array}{c}3.00 \pm 0.000 \\
\text { ST }\end{array}$ & $\begin{array}{c}3.00 \pm 0.000 \\
\text { ST }\end{array}$ \\
\hline $\begin{array}{l}\text { 6. Management } \\
\text { on short term } \\
\text { storage } \\
\text { condition, } \\
\text { transport tin } \\
\text { the field, and } \\
\text { storage of } \\
\text { produce } \\
\text { produce }\end{array}$ & $\begin{array}{c}2.99 \pm 0.036 \\
\text { ST }\end{array}$ & $\begin{array}{c}3.00 \pm .0 .000 \\
\text { ST }\end{array}$ & $\begin{array}{c}3.00 \pm 0.000 \\
\text { ST }\end{array}$ & $\begin{array}{c}2.97 \pm 0.111 \\
\text { ST }\end{array}$ & $\begin{array}{c}2.99 \pm 0.052 \\
\text { ST }\end{array}$ & $\begin{array}{c}3.00 \pm 0.000 \\
\text { ST }\end{array}$ & $\begin{array}{c}3.00 \pm 0.000 \\
\text { ST }\end{array}$ & $\begin{array}{c}2.98 \pm 0.097 \\
\text { ST }\end{array}$ & $\begin{array}{c}3.00 \pm 0.000 \\
\text { ST }\end{array}$ & $\left|\begin{array}{c}3.00 \pm 0.000 \\
\text { ST }\end{array}\right|$ & $\begin{array}{c}3.00 \pm 0.000 \\
\text { ST }\end{array}$ & $\begin{array}{c}2.99 \pm 0.041 \\
\text { ST }\end{array}$ & $\begin{array}{c}2.97 \pm 0.111 \\
\text { ST }\end{array}$ & $\begin{array}{c}2.99 \pm 0.076 \\
\text { ST }\end{array}$ & $\begin{array}{c}3.00 \pm 0.000 \\
\text { ST }\end{array}$ & $\begin{array}{c}2.98 \pm 0.055 \\
\text { ST }\end{array}$ & $\begin{array}{c}3.00 \pm 0.000 \\
\text { ST }\end{array}$ & $\begin{array}{c}2.99 \pm 0.035 \\
\text { ST }\end{array}$ & $\begin{array}{c}3.00 \pm 0.000 \\
\text { ST }\end{array}$ & $\begin{array}{c}2.97 \pm 0.115 \\
\text { ST }\end{array}$ \\
\hline $\begin{array}{l}\text { 7. Personal } \\
\text { hygiene } \\
\text { management }\end{array}$ & $\begin{array}{c}2.99 \pm 0.072 \\
\text { ST }\end{array}$ & $\begin{array}{c}2.97 \pm 0.075 \\
\text { ST }\end{array}$ & $\begin{array}{c}2.97 \pm 0.082 \\
\text { ST }\end{array}$ & $\begin{array}{c}3.00 \pm 0.000 \\
\text { ST }\end{array}$ & $\begin{array}{c}2.97 \pm 0.103 \\
\text { ST }\end{array}$ & $\begin{array}{c}2.98 \pm 0.055 \\
\text { ST }\end{array}$ & $\begin{array}{c}2.97 \pm 0.082 \\
\text { ST }\end{array}$ & $\begin{array}{c}3.00 \pm 0.000 \\
\text { ST }\end{array}$ & $\begin{array}{c}2.91 \pm 0.157 \\
\text { ST }\end{array}$ & $\begin{array}{c}3.00 \pm 0.000 \\
\text { ST }\end{array}$ & $\begin{array}{c}2.98 \pm 0.092 \\
\text { ST }\end{array}$ & $\begin{array}{c}2.99 \pm 0.041 \\
\text { ST }\end{array}$ & $\begin{array}{c}2.97 \pm 0.075 \\
\text { ST }\end{array}$ & $\begin{array}{c}2.99 \pm 0.038 \\
\text { ST }\end{array}$ & $\begin{array}{c}3.00 \pm 0.000 \\
\text { ST }\end{array}$ & $\begin{array}{c}2.98 \pm 0.055 \\
\text { ST }\end{array}$ & $\begin{array}{c}3.00 \pm 0.000 \\
\text { ST }\end{array}$ & $\begin{array}{c}2.98 \pm 0.078 \\
\text { ST }\end{array}$ & $\begin{array}{c}2.98 \pm 0.058 \\
\text { ST }\end{array}$ & $\begin{array}{c}2.98 \pm 0.058 \\
\text { ST }\end{array}$ \\
\hline $\begin{array}{l}\text { 8. Management of } \\
\text { data record and } \\
\text { traceability }\end{array}$ & $\begin{array}{c}2.98 \pm 0.079 \\
\text { ST }\end{array}$ & $\begin{array}{c}2.97 \pm 0.075 \\
\text { ST }\end{array}$ & $\begin{array}{c}3.00 \pm 0.000 \\
\text { ST }\end{array}$ & $\begin{array}{c}2.98 \pm 0.055 \\
\text { ST }\end{array}$ & \begin{tabular}{c|}
$2.96 \pm 0.112$ \\
ST
\end{tabular} & $\begin{array}{c}2.98 \pm 0.055 \\
\text { ST }\end{array}$ & $\begin{array}{c}3.00 \pm 0.000 \\
5 T\end{array}$ & $\begin{array}{c}2.99 \pm 0.049 \\
\text { ST }\end{array}$ & $\begin{array}{c}2.91 \pm 0.157 \\
\text { ST }\end{array}$ & $\left|\begin{array}{c}3.00 \pm 0.000 \\
5 T\end{array}\right|$ & $\begin{array}{c}2.98 \pm 0.092 \\
\text { ST }\end{array}$ & $\begin{array}{c}2.98 \pm 0.056 \\
\text { ST }\end{array}$ & $\begin{array}{c}2.97 \pm 0.075 \\
\text { ST }\end{array}$ & $\begin{array}{c}2.99 \pm 0.052 \\
\text { ST }\end{array}$ & $\begin{array}{c}3.00 \pm 0.000 \\
\text { ST }\end{array}$ & $\begin{array}{c}2.98 \pm 0.055 \\
\text { ST }\end{array}$ & $\begin{array}{c}3.00 \pm 0.000 \\
\text { ST }\end{array}$ & $\begin{array}{c}2.98 \pm 0.084 \\
\text { ST }\end{array}$ & $\begin{array}{c}3.00 \pm 0.000 \\
S T\end{array}$ & $\begin{array}{c}2.97 \pm 0.078 \\
\text { ST }\end{array}$ \\
\hline
\end{tabular}

Note: Rating scale of practice on GAP application in the NDMST Mango Production: 2.34-3.00 = Strongly (ST), 1.67-2.33 = Fair (FA), 1.00-1.66 = Poor (PO) 


\subsection{Production management and GAP application in the NDMST mango production for export by members of the BHME community enterprise}

The mango production of the BHME Community Enterprise was mostly on-season (or regularseason) followed by off-season, before-season and after-season (four season per year), respectively. The production met all eight provisions of the GAP standard module namely (1) water resources management (2) farm land and cultivation management (3) agricultural hazardous substances management (4) quality management in farming and before harvesting processes (5) quality management in harvesting and post harvesting processes (6) management on short term storage condition, transport in the field, and storage of produce (7) personal hygiene management and (8) management of data record and traceability (Table 2).

Based on the Cross-tab analysis concerning five areas of (1) members in community enterprise (year) (2) experience in mango production (year) (3) harvesting farm area (4) labour number and (5) mango production income, it was found that all areas were positively related to the meeting of the GAP standard, in accordance with research by Mahadlek et al. (2020), which reported that producing according to the GAP standard yielded higher amount and better quality of the produce. By following the GAP standard, the production became systematic, the use of fertiliser efficient, pest control kept under control and fruits subjected to bagging, as opposed to the general practice where the production was not systematic, fertilising was done sporadically and fruit bagging was not the norm; as a result, following the GAP standard allowed higher production in terms of both quality and quantity leading eventually to higher income (Mahadlek et al., 2020).

\subsection{Impact on and coping towards NDMST mango production for export by the BHME community enterprise and its members during the COVID-19 pandemic}

During the height of the spread of COVID-19, the export of the NDMST mango was heavily impacted. According to the severity of the impact and the corresponding response carried out by the enterprise, its members and export distributors, two periods were identified; the period of high severity (10-24 March 2020) and the period of lessened severity (25 March - 31 May 2020). During the covod19 outbreak, it was the same time for mango harvesting that farming on-season (or regular-season) (Figure 3 and 4).

\subsubsection{The impact on the members and their coping strategies of NDMST mango farmers in Khon} Kaen province during the pandemic of COVID-19

1) The period of highly severe impact with little room for adjustment - The initial period of adjustment to cope (10 - 24 March 2020)

Delaying harvest and prioritising marketing: The enterprise did not collect their produce and no processing took place. The members delayed their harvest with only a small amount of the produce harvested corresponding to the on-season yield of about $5 \%$. The produce was sold by individual members and instead of working in the farms, the members diverted their labour towards marketing in order to help with the sales of the mangos. In this initial period, they were able to sell 40 tonnes of mangos, an average of 0.7 tonne per farm (Figure 3 ).

2) The period of moderate severity with some ability for adjustment - The second period of adjustment to cope (25 March - 31 May 2020)

Having support from government and private agencies and able to sell some of the produce: The enterprise members were able to harvest the rest of the produce representing about $90 \%$ ( 775 tonnes) of the total amount of produce in that season (on average 14 tonnes per farm). $9.69 \%$ ( 79 tonnes) of the total mangos harvested were exported through the enterprise, $66.21 \%$ (540 tonnes) were sold in the domestic market and $14.10 \%$ (115 tonnes) were sold by the individual members (Figure 4).

The members of the BHME Community Enterprise were initially affected by the government measures to contain the spread of the virus. State of emergency was declared for all of Thailand and people were forbidden to leave their homes from 10:00 PM to 4:00 AM. General inter-province travel restrictions were put in place and international flights were temporarily prohibited from entering the country in March 2020 (Chanratchakit, 2020). Farmers lost their revenues through shrinking markets and 
falling prices (Songbanthit, 2020). At the height of the crisis, around half of Thai farmers were either in the middle of the plantation process or already had just harvested their agricultural products. Most of these farmers were in the upstream portion of the supply chain who exported or internally sold their produce. Because of the government measures in response to the COVID-19 crisis, disruption in transportation and the logistics as well as the change in demand could have a direct effect on the income of farmers (Chantarat et al., 2020). The produce harvested by the members were affected because the enterprise was unable to operate as usual, i.e., it could not collect and buy the produce. Moreover, export companies stopped buying the produce because they were not able to export them. After the initial period of severe impact, the community enterprise was helped with the purchase of the produce by both governmental and private agencies. Also, the produce could be distributed through an online channel. In comparison to the initial phase, the produce could be sold in higher amounts; however, because of the dumping of the price compared to that of the previous year, there was significant reduction in the working capital that would be needed to produce the NDMST mango in the next season.

\subsubsection{The impact and coping strategies of the community enterprise (Farmers institute)}

1) The period of highly severe impact with little room for adjustment - The initial period of adjustment to cope (10 - 24 March 2020)

The community enterprise not gathering the produce from its members and the enterprise being in contact with relevant agencies to solve the crisis: because export companies did not take the produce, the enterprise adjusted itself to the brunt of the pandemic by coordinating with governmental and private agencies for finding channels to distribute its produce and temporarily ceasing the processing of the produce. Efforts were put on marketing the products. As a result of the enterprise's coordination with the relevant agencies, it was provided help in terms of marketing, distributing and transporting the products within the domestic market (Figure 3).

2) The period of moderate severity with some ability for adjustment - The second period of adjustment to cope (25 March - 31 May 2020)

Able to export some of the produce with prices slashed by more than half: As a result of the export distributors' adjustment by using chartered flights for the export of the mangos, the enterprise could export $9.69 \%$ ( 79 tonnes) of their produce with prices slashed by $40 \%$ in comparison to the previous year, while $66.21 \%$ (540 tonnes) were sold in the domestic market with a price fall of $50 \%$ (Figure 4).

Cullen (2020) reported when the COVID-19 pandemic was raging through the globe, many countries closed their international borders in an attempt to contain the spread of the virus. Initially, there were sufficient amounts of goods for consumption; however, as the crisis dragged on, tension built up in the food supply chains and there were several problems that affected farmers, the production of the produce, processing factories, distribution as well as disruption of maritime transport and the logistics. This stood in contrast to the world food crisis of 2007-2008, where the supply could still be maintained. Trade restrictions were not only unnecessary but also had a severe impact on both the producer and the consumer. This caused panic in the market. For valuable goods that require labour instead of machinery, there needs to be a balance between the production and the labour. When countries were fighting the spread of COVID-19, at the same time, they were trying to maintain the food supply chain and enacting appropriate measures to ensure that the spread would not lead to a food crisis. The spread of the disease has impacted both the supply and demand causing reduction in the workforce within the agricultural sector (Gunjal \& Senahoun, 2016) and the industrial sector leading to a transition from cash crops to food crops (FAO \& UNAIDS, 2003), which in turn affects household incomes as well as food security (United Nations, 2004).

When the disease was spreading, Thailand's Kasikornbank (2020) assessed the impact and found that it would affect the country's industry in four ways: 1 ) the fall in the number of international tourists which was forecast to drop by $21.2 \%$ (8.3 million arrivals) compared to the previous year, 2 ) the export as a result of the disruption in demand from the international market, 3) production and investment particularly for those that relied on raw materials from China and 4) reduced household spending as a result of reduced spending by some people and the decrease in international tourist arrival, leading to income reduction (Kasikornbank, 2020). Food supply and food security were heavily affected because of limitations on liquidity and lower purchasing power. The impact was strongly felt in vulnerable 
population groups. Strict measures designed to contain the spread of the virus affected food production worldwide. Measures should protect not only the health of the population but also food security for the people reducing damage on the growth of the economy (Siche, 2020). Moreover, an analysis by BioThai (a non-government organization in Thailand) of the world and domestic markets found that agricultural products which serve as part of the industry's conveyor belt were heavily affected since the beginning of March. Particularly as the World Health Organization announced that COVID-19 was a pandemic, prices of agricultural products fell, exporting distributors temporarily put a stop to export contracts with monopoly on the production, control of agricultural systems and food supply chains (Bangkok Biz News, 2020).

\subsubsection{The impact on and the coping strategies of export distributors}

1) The initial period of heavy impact with little room for adjustment --The initial period of adjustment to cope (10 - 24 March 2020)

Exporting companies unable to buy produce from the enterprise and efforts put on finding ways to purchase produce for export: Some of the delivery systems were put on hold because of the lockdown measures to control the virus spread in some countries. However, the companies tried to find ways to transport the produce to help alleviate the impact on the farmers and to keep business contracts (Figure 3).

2) The period of moderate impact with an ability for some adjustment - The second period of adjustment to cope (25 March - 31 May 2020)

Export distributors exported some of the produce using chartered flights: Chartered flights could deliver $9.69 \%$ (79 tonnes) of the total produce to the international market, which was below a forecast of 300 tonnes. The enterprise and the farmers were asked to slash the price by $40 \%$ compared to the price of the previous year (Figure 4).

Laborde et al. (2020) reported that during the severe spread of the COVID-19 pandemic, there were attempts both to contain the spread of the virus and to avoid the occurrence of food crisis which would have a strong impact on the poor worldwide. Although serious shortages of food could be avoided, markets for agricultural products and foods were disrupted because of restrictions placed on movement of people and labour, the change in food demand as a result of the closing of restaurants and schools, and the loss of income. The pandemic has affected food security in four ways 1) preparedness (whether there is sufficient food), 2) access (whether people have enough food for consumption), 3) utilisation (whether people has sufficient nutrients) and 4) security (whether people can consume food when they want). The spread of COVID-19 has had a severe impact on access to food. People have opted to food of lower price, quality and nutritional values. There has been uncertainty in food prices. All these factors would constitute as a threat to food security. The health crisis should then be prevented from leading to a world food crisis. Urban centres have borne the brunt of the effect, risking to suffer multiple crises at the same time. Similarly, Pulighe \& Lupia (2020) stated that the severity of the pandemic and the lockdown imposed by many different countries had led people to realise the importance of food for urban populations. The closing of international borders as well as restrictions on transport on the produce cause losses to food and export costs, particularly to vegetable or other produce that quickly spoil from non-self-sufficient countries. Agriculture in cities of developed countries should incorporate the idea of green infrastructure (GI) by for example promoting hydroponics, aeroponics, aquaponics and rooftop greenhouses. 


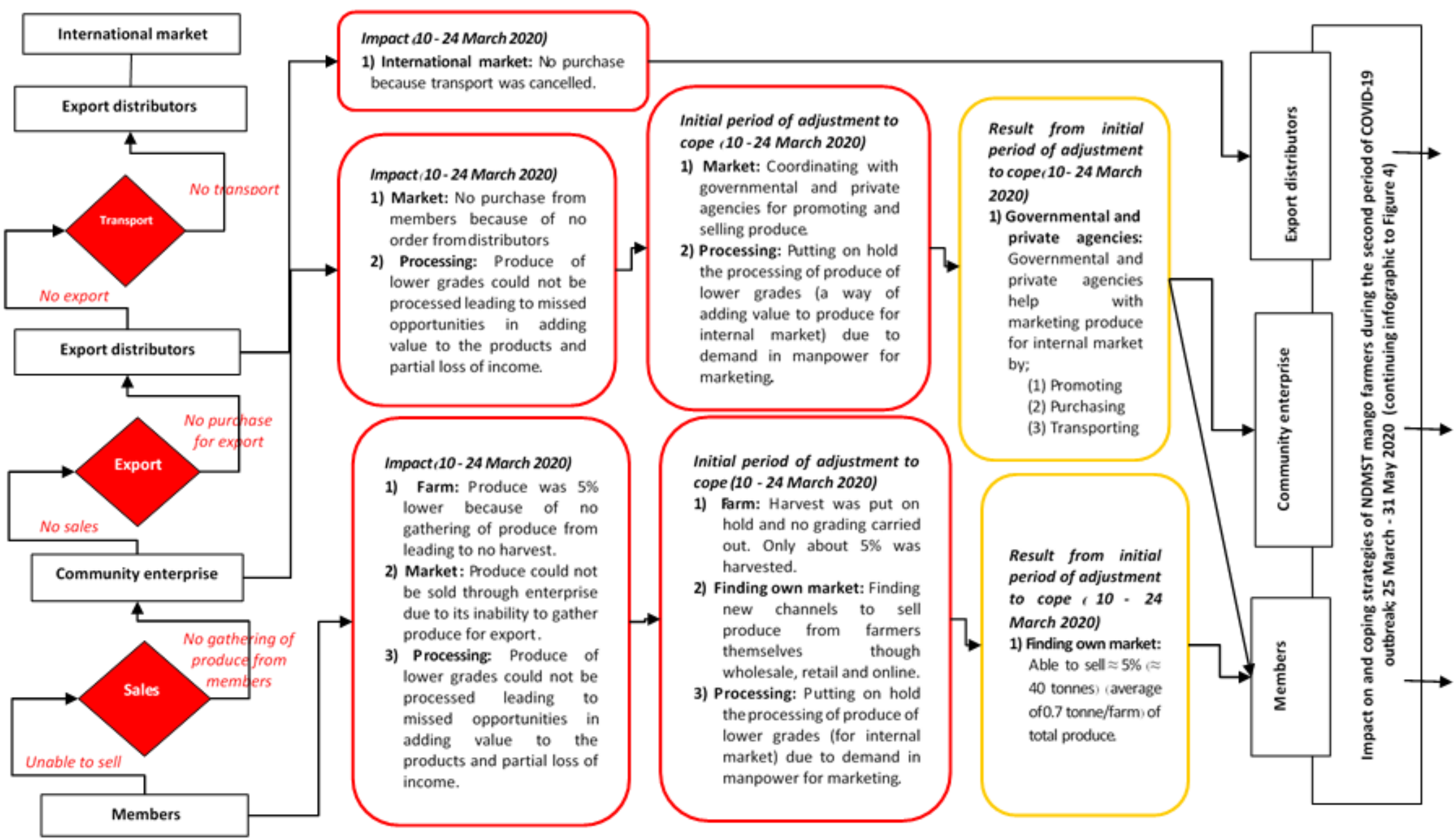

Figure 3. Impact on and coping strategies of NDMST mango farmers during the initial period of COVID-19 outbreak; $10-24$ March 2020 (it was the same time for mango harvesting that farming on-season (or regular-season)) 


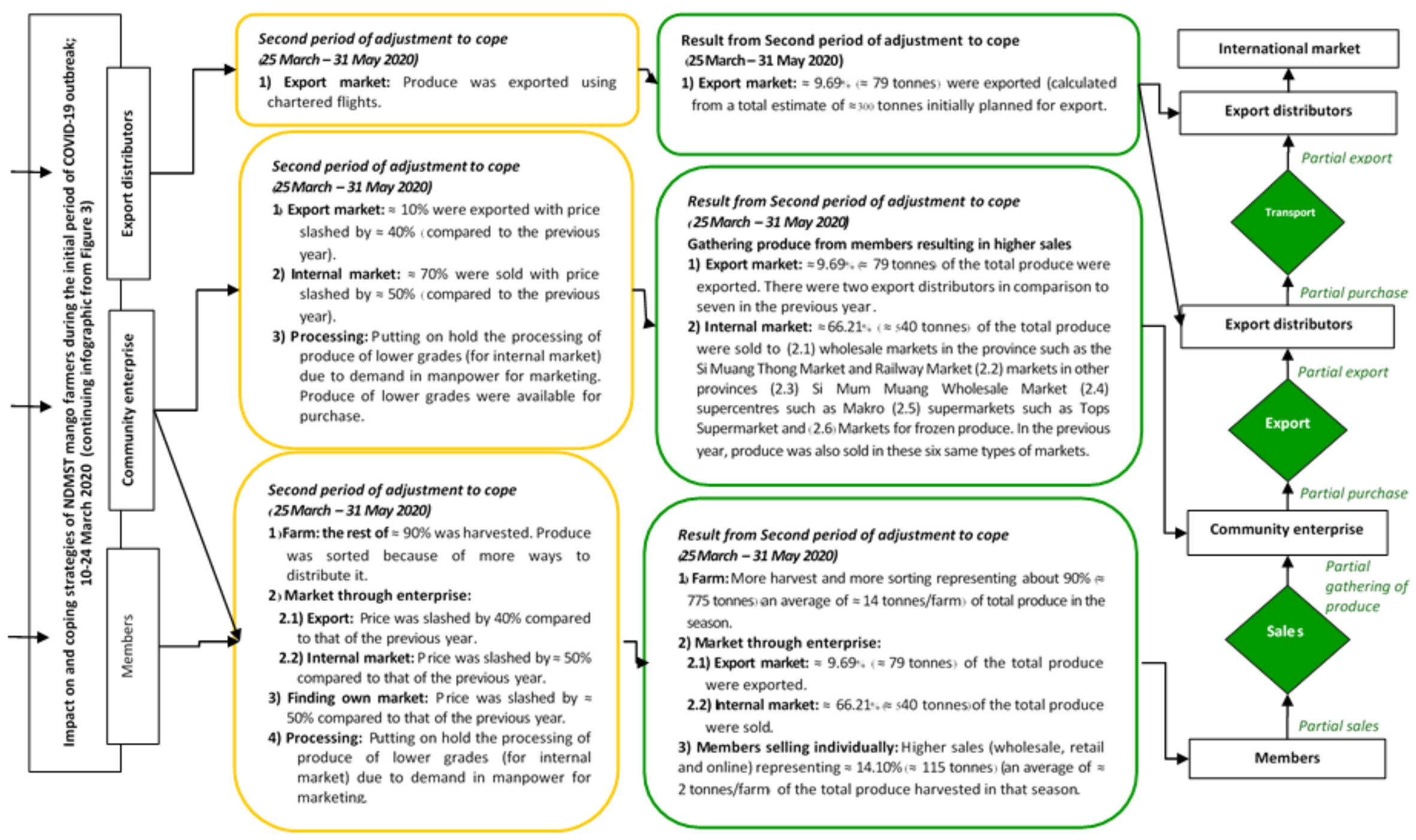

Figure 4. Impact on and coping strategies of NDMST mango farmers during the second period of COVID-19 outbreak; 25 March - 31 May 2020 (it was the same time for mango harvesting that farming on-season (or regular-season)) 
After the severity of the pandemic had alleviated in the month of April 2020, the Office of Agricultural Affairs and Bureau of Foreign Agricultural Affairs (2020) reported that agricultural produce could partly be exported with major markets being China, Japan, South Korea and Australia and USA. Due to the high prices of certain Thai fruits and the consumers' choice of opting for essential goods, uncertainty in the demand remains. If the crisis deepens, consumers will have a tendency to change their consumption habits by refraining from making purchases from supermarkets or retail shops and instead turning to online shopping. Measures to cope with such pandemics would be to closely monitor the situation of the spread and enact appropriate measures to contain it, to find new distribution channels or new markets for the produce, and to promote the processing of fruits for example by freezing to preserve them for a longer period of time. Thailand has experienced the economic consequences of the spread of the avian influenza. Tangtaweewipat et al. (2005) reported the practice of poultry culling to stop the spread of the bird flu virus leading to reduced production of poultry. This caused farmers both on the small and medium scales to close their farms. Some consumers chose not to consumer poultry which greatly affected farmers, processing factories and distribution companies, leading to a significant reduction in incomes. Ruchiseesarod (2014) found that the spread of the bird flu affected the consumer confidence by as much as $50 \%$. Because of their incomplete understanding of the bird flu, consumption of chicken declined and consumers took extra precaution when choosing the meat to buy. In the year 1997, during the Asian Financial Crisis, also known as the Tom Yam Kung Crisis, Mahasinpaisan (2017) and the Secretariat of the House of Representatives (2017) reported that it was the most severe financial crisis to befall the country in modern history affecting financial institutions, entrepreneurs as well the general public who suffered from inflation, elevated prices of goods and high unemployment. According to the Thai PBS News (2020), the origin of the Tom Yum Kung crisis in Thailand was the economic imbalance. This was different from the current crisis of COVID-19 where the cause of the crisis was transmission and spread of the virus. Health authorities throughout the world have been busy with vaccine development to put an end to the crisis. Measures announced by governments and central banks during the current crisis have been for the purpose of sustaining the economic mechanisms, while the 1997 measures aimed to resuscitate the economic mechanisms.

\section{Summary and Recommendation}

Prior to the spread of COVID-19, we studied the operational conditions of the BHME Community Enterprise, which produced the NDMST mango for export and found that the community enterprise and its members were provided support in terms of promoting and developing the production from the Farmers Institute, Farmer Leader and relevant agencies so that they could meet the production standards of Good Agricultural Practices (GAP). By following the GAP standard, they were able to control the quality of the product and manage their operation with efficiency. The main export markets were Japan, South Korea and China with mangos graded 3A or 2A exported. Those with lower grades were sold in the domestic market through middlemen who sorted the mangos into grade 0,1 and 2 . Mangos of premium quality (those with the same grades as the mangos destined for the international market) were also packaged and sold in the domestic market. The sales were conducted through the enterprise or by the individual members. Some of the produce was processed to add value to it and sold in the domestic market, serving as a way to promote the enterprise and its members.

The mangos for export were mostly on-season followed by off-season, before-season and afterseason, respectively. Good agriculture was practised in all areas and according to our Crosstab analysis of five areas, we their found positive relationship with the GAP.

As a consequence of the spread of COVID-19, the initial period (10-24 March 2020) was harsh for the community enterprise and its members. There was little they could do to adjust to the severity of the pandemic because export distributors could not purchase mangos from them. The members harvested the produce and sold it themselves. The enterprise coordinated with governmental and private agencies to find new channels for distribution. During the second period ( 25 March -31 May 2020), the severity of the impact was lessened and they had more room for adjustment. By coordinating with the relevant agencies, they were able to sell some of the produce. The price of the produce sold in the domestic market was slashed by $50 \%$ in comparison to that of the previous year. Export could be 
revived through transportation by chartered flights with the price down by about $50 \%$ compared to that of the previous year. Only 79 tonnes were exported, way below their pre-COVID-19 estimate of 300 tonnes.

The BHME Community Enterprise, along with its members and relevant agencies, could benefit greatly from joint discussion and conclusion on strategies related to the planning and marketing the produce. Were crises similar to the current COVID-19 pandemic to occur in the future, they would be able cope, through lessons learned together from the ongoing crisis, with the impact on export and distribution of the produce.

Acknowledgments: Thanks to key informants and farmers who provide information that is useful in this research. Thanks to Faculty of Agriculture, Khon Kaen University who supported of this research.

Conflicts of interest: The authors declare no conflict of interest.

Ethical statement: We declare that this manuscript is original, has not been published before, and is not currently being considered for publication elsewhere. We wish to confirm that there are no known conflicts of interest associated with this publication and there has been no significant financial support for this work that could have influenced its outcome. Also, we further confirm that the manuscript has been read and approved by all named authors and that there are no other persons who satisfied the criteria for authorship but are not listed. We confirm that any aspect of the work covered in this manuscript does not contain any studies with experimental design by using animals or humans as subjects performed by any of the authors. Lastly, we care and protects the pandemic of COVID-19 during research activity in the study site.

\section{References}

Bangkok Biz News. (2020). The impact of CovidCOVID-19 disease on agriculture. Retrieved May 8 , 2020, from https://www.bangkokbiznews.com/news/detail/874569

BBC NEWS Thai. (2020). Coronavirus Disease: World Bank expects the impact of CovidCOVID-19 disease drag Thailand economy down second largest in Asia-Pacific. Retrieved May 8, 2020, from https://www.bbc.com/thai/international-52101949

Blum, S., Brow, M., Silver, R.C. (2012). Coping. In Ramachandran, V. S. (Ed.) Encyclopedia of Human Behavior (Second Edition). Academic Press.

Borisutdhi, Y. (2015). Community Study: Perspective Based on Research and Scientific Process. Kaen University Printing House.

Bureau of General Communicable Diseases. (2019). Avian Influenza. Department of Disease Control Ministry of Public Health. Retrieved May 8, 2020, from http://dcd.ddc.moph.go.th/uploads/file/EID/AI_Ebola_MERs_July2019.pdf

Centre for Studies on Human Stress (CSHS). (2019). Coping Strategies. Retrieved January 4, 2021, from https://humanstress.ca/stress/trick-your-stress/steps-to-instant-stress-management/

Chanratchakit, I., Laongmanee, P., Lanmeen, J., Suasi, T., Sornkliang, J., Tiaye, Yasook, N., \& Putsa, S. (2020). Special report on the impact of the COVID-19 crisis How the local fishermen are adapting. Samut Prakan: Southeast Asian Fisheries Development Center/Training Department.

Chantapong, S., \& Tonghui, T. (2020). The World and Thailand Economy after CovidCOVID-19: Diseases revolutionize the world to new life in the future. Bank of Thailand. Retrieved July 19, 2020, from https://www.bot.or.th/Thai/BOTMagazine/Pages/256303_CoverStory.aspx

Chantapong, S., Tonghui, T., Bhula-or, R., \& Chimmamee, M. (2020). COVID-19: Social Distancing and Population migration from the sociological: Stay at home and stop the spread of the virus for the sake of the nation. Bank of Thailand. Retrieved July 17, 2020, from https://www.bot.or.th/Thai/ResearchAndPublications/articles/Pages/Article_31Mar2020.aspx

Chantarat, S., Lamsam, A., Adulthananusak, N., Rattanawararak, L., Rittinon, C., \& Sangiamnet, B. (2019). The farmers households of Thailand in the COVID-19 crisis. Making Research Accessible, 2020(11), 1-12.

Cheewatrakoolpong, C. (2020, March 23). The impact of Covid-19 disease on global economic chain. Chulalongkorn University. Retrieved May 14, 2020, from https://www.chula.ac.th/cuinside/28614 
Cullen, M. T. (2020, March 29). COVID-19 and the risk to food supply chains: How to respond?. Food and Agriculture Organization of the United Nations. Retrieved July 14, 2020, from http://www.fao.org/documents/card/en/c/ca8388en

Danaisawat, T., \& Hengpraprom, S. (2018). Emerging infectious disease from bats. Veterinary Integrative Science, 16(3), 135-144. Retrieved from https://he02.tcithaijo.org/index.php/vis/article/view/151979

Dangbudda, N. (2011). Key Success of Banhad Community Mango Enterprise in Banhad Tambon, Khon Kaen Province. An Independent Study Report for the Degree of Master of Public Administration in Local Government, College of Local Administration, Khon Kaen University.

Department of Disease Control. (2020). Coronavirus Disease 2019. Ministry of Public Health. Retrieved July 14, 2020, from https://ddc.moph.go.th/viralpneumonia/file/g_km/handout001_12032020.pdf

Food and Agriculture Organization of the United Nations \& The Joint United Nations Programme on HIV/AIDS. (2003). Addressing the impact of HIV/AIDS on ministries of agriculture: focus on eastern and southern Africa. Retrieved July 18, 2020, from http://www.fao.org/3/ad508e/ad508e0a.htm

Food and Agriculture Organization of the United Nations. (2020). Agri-food markets and trade policy in the time of COVID-19. Retrieved July 14, 2020, from http://www.fao.org/documents/card/en/c/ca8446en

Gopinath, G. (2020, April 14). The Great Lockdown: Worst Economic Downturn Since the Great Depression. International Monetary Fund. Retrieved July 15, 2020, from https://blogs.imf.org/2020/04/14/the-great-lockdown-worst-economic-downturn-since-the-greatdepression

Gunjal, K., \& Senahoun, J. (2016). Assessing the impact of infectious disease outbreaks on agriculture and food security: The case of the Ebola virus disease outbreak in West Africa. Food and Agriculture Organization of the United Nations. Retrieved July 18, 2020, from http://www.fao.org/3/a-i5641e.pdf

Jumroensarn, J. (2010). Marketing management of Off-Season Mango: a case study of Banhad Community Enterprise, Banhad District, Khon Kaen Province. An Independent Study Report for the Master of Science in Agribusiness, Graduate School, Khon Kaen University.

Kasikornbank. (2020). K-Bank reflections on market, the impact on Covid-19 pandemic expected the Thailand Monetary Policy Committee (MPC) to cut interest rates 2 more times this year. Kasikornbank financial markets views and analysis: capital market business, March 5, 2020. Retrieved March 5, 2020, from https://www.kasikornbank.com/en/business/Foreign-ExchangeMarket/KBankCapitalMarketPerspective/ KBank\%20Reflections\%20on\%20Markets\%20-\%20ผลกระทบ\%20Covid$19 \% 20$ คาดทำให้\% 20 กนง. $\% 20$ ลดดอกเบี้ยอีก\% $202 \% 20$ ครั้งในปีนี้.pdf

Laborde, D., Martin, W., Swinnen, J., \& Vos, R. (2020). COVID-19 risks to global food security. Science, 369(6503), 500-502. https://doi.org/10.1126/science.abc4765

Mahadlek, J., Chainet, J., \& Kwunsiriwong, S. (2019). Productivity comparison of Namdokmai Sithong Mango by using Good Agricultural Practice (GAP) and general agriculture execute in Sao Cha-ngok, Bangkhla District, Chacoongsao Proviemce. PSRU Journal of Science and Technology, 4(2), 69-84.

Mahasinpaisan, T. (2017). From Tom Yum Kung Crisis to Thailand 4.0. Panyapiwat Journal, 9 (Special Issue), Editorial.

Ministry of Public Health. (2011). Knowledge (Factsheet) on Emerging Infectious Diseases. Bangkok: The War Veterans Organization of Thailand Under Royal Patronage of His majesty the King.

Office of Agricultural Affairs and Bureau of Foreign Agricultural Affairs. (2020, March 1). Information for preparing measures to the impacts on fruit exports in Thailand from the situation of COVID-19 outbreak. Retrieved May 8, 2020, from https://www.moac.go.th/foreignagri-news-files421091791421

Paediatric Infectious Disease Society of Thailand. [n.d.]. CME: Cholera... The disease comes with food and water. Retrieved May 13, 2020, from https://www.pidst.or.th/A463.html 
Pulighe, G., \& Lupia, F. (2020). Food first: COVID-19 outbreak and cities lockdown a booster for a wider vision on urban agriculture. Sustainability, 12(12), 5012. https://doi.org/10.3390/su12125012

Ruchiseesarod, C. (2014). The Effect Avian Influenza (Bird Flu) on Information Perception Chicken Purchase and in Bangkok. Veridian E-Journal Science and Technology, 1(5), 1-10.

Siche, R. (2020). What is the impact of COVID-19 disease on agriculture?. Scientia Agropecuaria, 11(1), 3-6. http://dx.doi.org/10.17268/sci.agropecu.2020.01.00

Songbanthit, S. (2020, May 26). The impact of Covid-19 disease on agriculture, proposals and farmers adaptation. Local Act. Retrieved July 19, 2020, from https://www.landactionthai.org/2012-05-18-03-2445/article/item/2393-19-2.html

Srinil, M. (2020, May 4). The impact Covid-19 disease on mango farmers selling cheaply, at 30 Baht in Thailand. DOKBIA ONLINE. Retrieved July 17, 2020, from https://www.dokbiaonline.com/post/mango

Tangtaweewipat, S., Cheva-Isarakul, B., Ruangwong, J., Saengdoaruang, S., Suna, C., \& Songkun, T. (2005). The Impact of Avian Influenza Epidemic and the Restoration Measurements: Production Sectors. Proceedings of 44th Kasetsart University Annual Conference: Animal, Veterinary Medicine, Bangkok.

Thai PBS News. (2020, April 16). The difference between COVID-19 disease and Tom Yum Kung Crisis. Retrieved June 21, 2020, https://news.thaipbs.or.th/content/291266

Thailand Science Research and Innovation. (2020, April 14). TSRI support farmers through the COVID19 crisis for export of mango shipping to Japan and South Korea. Retrieved July 17, 2020, from https://www.mhesi.go.th/home/index.php/pr/news/1380-19-7

The secretariat of the house of representatives. (2017, August) Lessons on the Tom Yum Kung crisis from Economy of Thailand and ASEAN region. Retrieved Jube 25, 2020, from https://library2.parliament.go.th/ejournal/content_af/2560/aug2560-1.pdf

Thongcharoen, P. (2009a). World-shaking outbreaks 2. Augsornsamai Printing House.

Thongcharoen, P. (2009b). World-shaking outbreaks 7. Augsornsamai Printing House.

United Nations. (2004). The Impact of AIDS. Retrieved July 18, 2020, from https://www.un.org/en/development/desa/population/publications/pdf/hiv/impact/chap5.pdf 